\title{
Premature ventricular complex-induced chronic cough and cough syncope
}

\author{
S. Stec*, M. Dąbrowska\#, B. Zaborska*, P. Bielicki”, M. Maskey-Warzęchowska”, \\ W. Tarnowski ${ }^{\oplus}$, R. Chazan ${ }^{\#}$ and P. Kulakowski*
}

ABSTRACT: The present case study reports a case of chronic cough and cough syncope associated with frequent premature ventricular complexes (PVCs).

Careful analysis of cough-related symptoms and ECG monitoring led to the suspicion of PVCinduced cough. A coincidence between PVCs and episodes of cough was also documented by a portable multichannel recorder. Moreover, Doppler echocardiography revealed a PVC-induced transient increase in the pulmonary artery blood flow.

After exclusion of other possible aetiologies, complete relief of chronic cough and cough syncope was achieved by radiofrequency ablation of the arrhythmogenic focus located in the right ventricular outflow tract.

Premature ventricular complexes should be considered as a cause of chronic cough and cough syncope and an interdisciplinary cooperation can lead to successful diagnosis and treatment of this condition.

KEYWORDS: Arrhythmias, cardiovascular disease, chronic cough, complication, symptom evaluation and management, syncope

hronic cough, defined as a cough persisting for $\geqslant 8$ weeks, has been reported to be a common symptom for which outpatient care is sought $[1,2]$. A number of management strategies have been investigated for chronic cough; however, even after a highly effective clinical workout, up to $7 \%$ of patients are discharged with a diagnosis of "unexplained cough" [2-5]. While it has been known for many years that cough can cause brady- and tachyarrhythmias [1], it has not been previously reported that a rhythm disturbance can also cause cough and lead to the life-threatening complication of syncope. The present case study reports a case of chronic cough and cough syncope associated with frequent premature ventricular complexes (PVCs).

\section{CASE REPORT}

A 36-yr-old female was referred for clinical evaluation because of chronic cough associated with mild palpitations and recurrent coughrelated syncope. The patient had a 9-month history of cough, without any previous respiratory infection or other conditions that might have caused the cough. Despite smoking cessation 2 months earlier, the cough persisted and the patient suffered from cough-related syncope every 2-3 weeks.

The patient reported attacks of nonproductive cough that clustered for 60-150 min every day. She denied any shortness of breath, symptoms of rhinitis, sinusitis or gastro-oesophageal reflux disease. The cough was her principal presenting symptom that significantly decreased her quality of life.

The patient presented no abnormality on physical examination; her pulse was regular and her body mass index was $23 \mathrm{~kg} \cdot \mathrm{m}^{2}$. Several measurements revealed a tendency to hypotension with blood pressure $90 / 60 \mathrm{mmHg}$, but with normal blood pressure values during orthostatic stress.

The patient's ECG was normal, however, during attacks of cough, an irregular pulse was noted and ECG revealed sinus rhythm with frequent unifocal PVCs of the left bundle branch block-like morphology and inferior axis.

Repeated 24-h Holter monitoring showed normal sinus rhythm with 800-2,500 PVCs, with various coupling intervals clustered for only $1-3$ h during daily activity. PVCs were recorded simultaneously

\section{AFFILIATIONS}

*Postgraduate Medical School,

Depts of Cardiology, and

"General and Gastroenterological

Surgery, Grochowski Hospital, and

\#Warsaw Medical University, Dept of Internal Diseases, Pneumology and

Allergology, Warsaw, Poland.

CORRESPONDENCE

P. Kulakowski

Postgraduate Medical School

Dept of Cardiology

Grochowski Hospital

Grenadierow 51/59 Street

04-073 Warsaw

Poland

Fax: 48228711108

E-mail: smstec@wp.pl

Received:

May 102006

Accepted after revision:

January 102007

\section{SUPPORT STATEMENT}

The study was supported in part by research grant no. 501-2-1-10-72/ 04 from the Postgraduate Medical School, Warsaw, Poland.

STATEMENT OF INTEREST

None declared.

European Respiratory Journal Print ISSN 0903-1936 Online ISSN 1399-3003 
with attacks of cough, as reported by the patient and event chart notes. No sustained or nonsustained ventricular tachycardia, or conduction abnormalities were noted. Numerous tests were performed to induce PVCs (exercise stress test, mental stress tests, sustained isometric handgrip manoeuvre, Valsalva manoeuvre and carotid sinus massage), however, all were ineffective. Short periods of PVCs associated with induction of cough were observed repeatedly only after intravenous infusion of a low dose of isoproteronol.

Standard echocardiography revealed mild mitral prolapse with small mitral regurgitation. Diameters of cardiac chambers, wall thickness and contractility were normal. Ejection fraction was $69 \%$. No main pulmonary artery abnormalities were observed. Moreover, the blood flow velocities in both ventricular outflow tracts (left and right ventricular outflow tract (LVOT and RVOT, respectively)) were analysed during PVCs by pulsed Doppler echocardiography. Mean (from four beats) stroke volumes (SVs) for left ventricle and right ventricle were calculated based on velocity time integral (VTI), and LVOT and RVOT diameters. As compared with sinus beats, PVCs with a $530 \mathrm{~ms}$ coupling interval decreased VTI and SVs measured in LVOT by $\sim 25 \%$ (from 30.1 to $22.2 \mathrm{~cm}$ and from 68.3 to $50.4 \mathrm{~mL}$ ). In LVOT, PVCs did not significantly change VTI when they were presented with a long coupling interval (650 ms; fig. 1a). In RVOT, mean peak flow velocity (measured from four sequences) during PVCs with a long coupling interval (650 ms) increased significantly as compared with sinus beat and post-PVC sinus beat $(1.17 \pm 0.053,0.89 \pm 0.067$ and $0.89 \pm 0.046 \mathrm{~m} \cdot \mathrm{s}^{-1}$, respectively; $\left.\mathrm{p}<0.001\right)$. Moreover, VTI $(23.0 \mathrm{~cm})$ and SV $(65.1 \mathrm{~mL})$ during PVC was augmented by $\sim 20 \%$ as compared with measurements obtained in sinus beats (18.0 $\mathrm{cm}$ and $50.9 \mathrm{~mL}$, respectively; fig. 1b). In RVOT, PVCs with a shorter coupling interval $(530 \mathrm{~ms})$ resulted in a nonsignificant decrease of VTI and SV in PVCs and in

a)
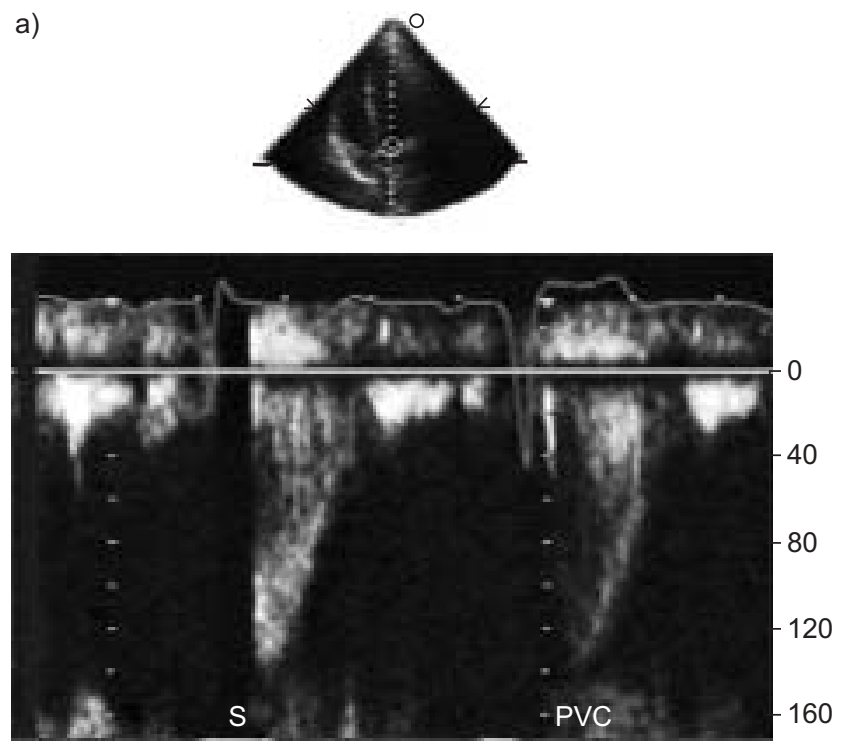

post-PVC beat. No reverse flow to pulmonary veins was observed during PVCs.

During 2 weeks of monitoring by an external event recorder, the patient registered episodes of ventricular bigeminy, which were associated with pre-syncopal episodes and cough. Subsequent treatment with verapamil, metoprolol and propafenone was ineffective.

Before electrophysiological study, the patient was referred for pulmonary investigation to exclude other causes of chronic cough. Spirometry and chest radiogram were normal, and a bronchial hyperreactivity test (histamine challenge) was negative. The concentration of total immunoglobulin E was within the normal range and skin prick tests with common inhaled allergens were negative. There was $<1 \%$ of eosinophils in induced sputum. Gastro-oesophageal reflux disease was excluded by negative oesophageal 24-h $\mathrm{pH}$ monitoring with an event recorder. No other abnormalities, especially implying upper airway cough syndrome, were found on laryngological examination, videolaryngoscopy and computed tomography scans of the paranasal sinuses.

A coincidence between PVCs and episodes of cough was documented by a portable multichannel recorder developed for sleep breathing disorders (HypnoPTT; Tyco Healthcare, Gosport, UK). Sudden changes in chest movements and flow through nasal cannula corresponding to episodes of cough were observed directly after certain episodes of PVC (fig. 2).

Finally, the patient was referred for invasive electrophysiological study and radiofrequency (RF) ablation. Programmed ventricular stimulation was negative. Due to a lack of spontaneous PVC activity, pace mapping and activation mapping were performed based on PVCs induced after isoproterenol infusion. The earliest ventricular endocardial

b)
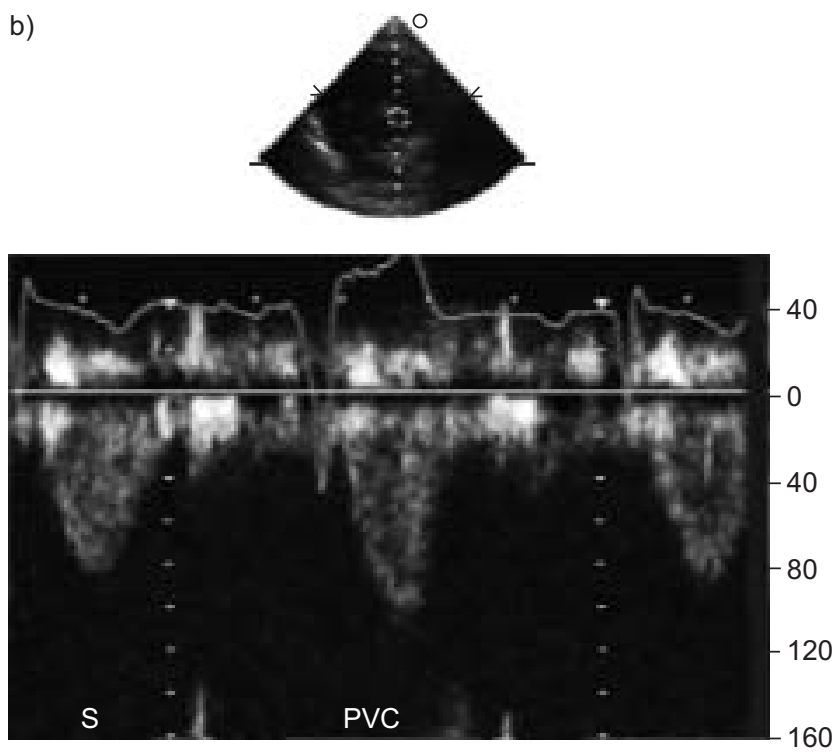

FIGURE 1. Pulsed Doppler recordings of blood flow in pulmonary and aortic valves during sinus rhythm and premature ventricular complexes (PVCs) with a long coupling interval $(650 \mathrm{~ms})$. a) Flow velocity pattern, velocity time integral (VTI) measurement and stroke volume (SV) calculation from the aortic valve (left ventricular outflow tract (OT)). b) Flow velocity pattern from pulmonary valve (right ventricular OT). PVC-associated augmentation in VTI and SV is visible in PVCs only in the right ventricular OT. $\mathrm{S}$ : sinus beat 


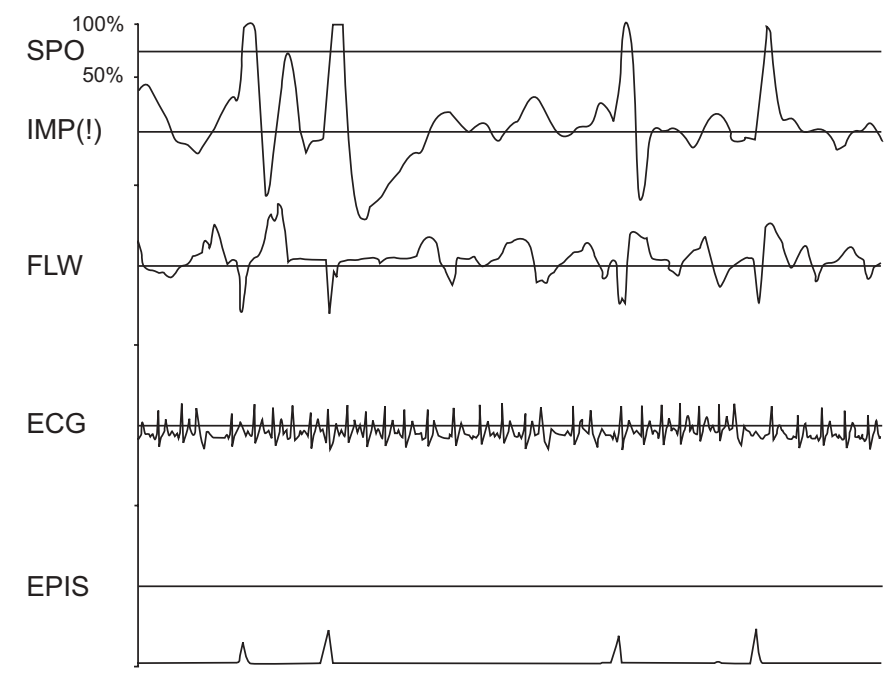

FIGURE 2. Multichannel monitoring system (HypnoPTT; Tyco Healthcare, Gosport, UK) shows sudden changes in chest movements (IMP) and air flow through the nasal cannula (FLW), observed just after premature ventricular complex (PVC). SPO: blood oxygen saturation; EPIS: episodes of coughing associated with PVCs (ECG tracing).

activation time of the PVCs (45 ms earlier than that on surface ECG) was localised at the posterior area of the RVOT (fig. 3a). After the fifth pulse of RF energy, PVCs completely disappeared and were no longer induced by infusion of isoproterenol and post-ablation pacing (fig. 3b).

The patient showed immediate improvement of symptoms after the procedure. She remained free of PVC-related symptoms, paroxysmal coughing and episodes of syncope during a 12-month follow-up. A 24-h ECG Holter recording obtained 4 weeks and 6 months after RF ablation showed two asymptomatic single PVCs and no PVCs, respectively.

\section{DISCUSSION}

While it was known that cough can cause arrhythmias, it has not been commonly reported that a rhythm disturbance can also cause cough or cough syncope. Many diseases and clinical conditions can cause chronic cough [1-5]. The most common causes of chronic cough in nonsmokers with normal chest radiographs are upper airway cough syndrome, asthma, nonasthmatic eosinophilic bronchitis and gastro-oesophageal reflux disease $[3,4]$.

It can be speculated that pulmonary trunk distension may trigger cough reflex by stimulating sympathetic innervation in RVOT or pulmonary artery. Although an increase in peak flow velocity in RVOT associated with PVCs was significant in the present study, it might have caused only a low increase in pressure in the pulmonary trunk. The cough reflex is initiated by irritating cough receptors primarily localised in the epithelium of the upper and lower respiratory tract. Afferent and efferent nerves in the vagus mediate the reflex [6]. The cough reflex in humans and complex mechanisms of integration of afferent nerve input in central nervous system needs further investigation [6].

From the present authors' large group of patients with PVCs, the patient presented herein was the only one with a)

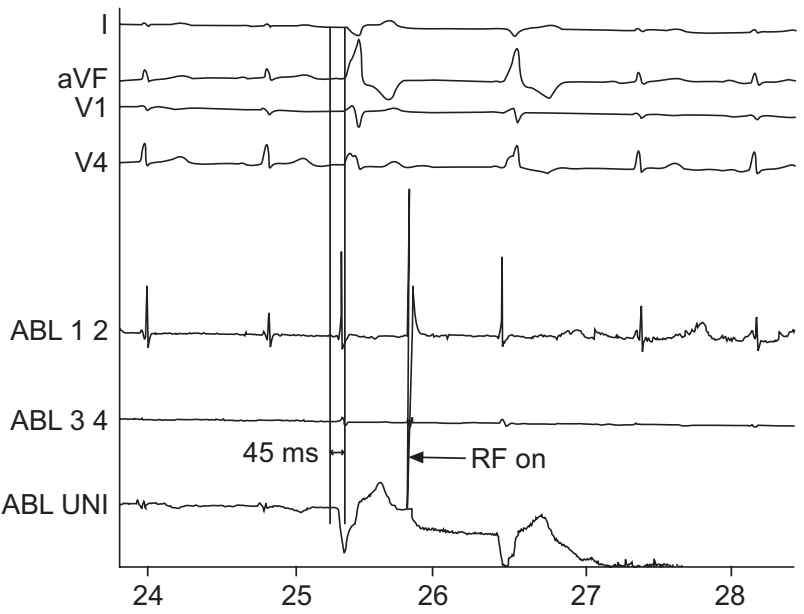

b)

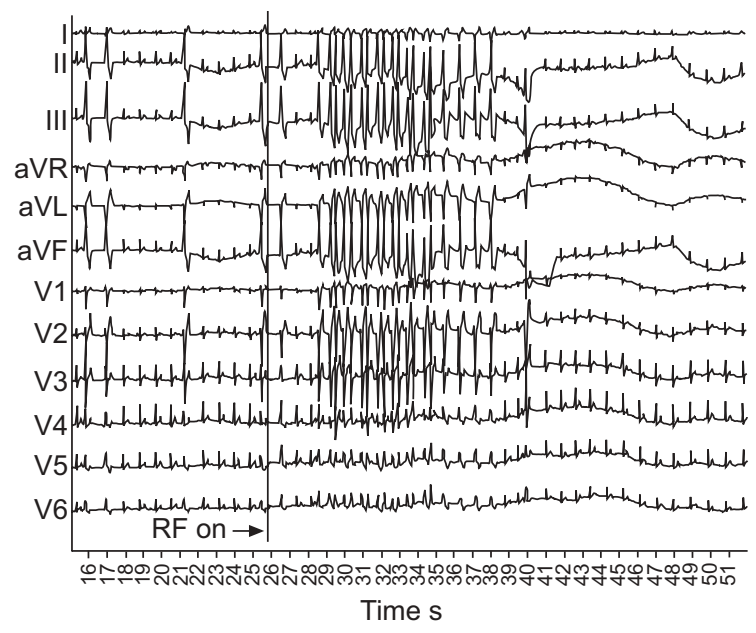

FIGURE 3. a) Activation mapping just before successful radiofrequency (RF) application. Surface ECG leads I, aVF, V1 and V4, as well as intracardiac recordings from the ablation electrode, are displayed (ABL 12 : bipolar signal from the tip of ablation electrode; $\mathrm{ABL} 34$ : bipolar signal from the proximal ablation electrode; $\mathrm{ABL}$ UNI: unipolar signal from ablation electrode). The onset of premature ventricular complexes (PVCs) in ABL 12 and ABL UNI preceded the beginning of PVCs on surface ECG by $45 \mathrm{~ms}$, which, together with the morphology of unipolar electrocardiogram, indicates a suitable site for RF energy delivery. The focus was localised in the postero-septal wall of right ventricular outflow tract, just below the pulmonary valve. RF on: beginning of RF ablation. b) Twelve-lead ECG showing sinus rhythm and left bundle branch-like morphology PVCs with inferior axis. RF energy delivery in the target site (RF on) leads to the induction of nonsustained ventricular tachycardia (nsVT) of the QRS morphology identical to clinical PVCs. After 15 QRS complexes, the rate of nsVT slows down, and is followed by complete elimination of the arrhythmia.

PVC-induced augmentation in pulmonary blood flow and was also the only one in whom cough was the main presenting symptom [7]. Reflex cough associated with post PVC-induced haemodynamic changes in pulmonary blood flow has been also reported by NIMII et al. [8]. Augmentation in blood flow following PVCs was associated with cough reflex after all PVCs, but in the present case, this association was documented less frequently, probably because of various coupling intervals of PVCs [8]. 
A similar observation between cough and irregular heart rhythm was reported by ODEH and OLIVEN [9]; however, they identified atrial premature complexes, and not PVCs, as factors inducing chronic cough. ZHOU et al. [10] reported that the hyperactive state of the major sympathetic nerve innervating the proximal pulmonary artery and RVOT may produce a discrete arrhythmogenic focus that is subject to neurohormonal regulation. Ablation of the sympathetic nerves innervating RVOT may provide a new approach to eliminate arrhythmogenic focus and its association with sympathetic nervous system [10]. A capsaicin or citric acid cough provocation test was not performed in the present patient's case; therefore, the sensitivity of cough reflex and its mechanism could not be evaluated further.

In the present case, the authors were able to demonstrate a novel application of the HypnoPTT system for differential diagnosis of cough. Simultaneous recording of air flow in the upper airways, sudden changes in chest movements and ECG helped to detect and associate cough with arrhythmia. HypnoPTT is usually applied in the preliminary tests for obstructive sleep apnoea syndrome, but in the present case, recording was performed for $\sim 5 \mathrm{~h}$ during daily rest in a supine or sitting position.

Various mechanisms have been reported to be associated with cough syncope [11-13]. The present authors suspect that the impairment of cerebrovascular blood flow during PVCs and hypotension could contribute to the development of syncope during frequent PVCs and uncontrolled paroxysm of vigorous coughing [7, 13, 14].

PVCs in patients without structural heart disease are benign; however, they may lead to a significant decrease in the quality of life and very serious conditions, such as tachycardiainduced cardiomyopathy or post-syncopal injury. RF ablation in symptomatic patients with idiopathic ventricular arrhythmias seems to be very effective and safe [15-18].

In conclusion, such a rare cause of chronic cough may be worth considering, as sometimes it may take up to 15 yrs from the onset of symptoms to diagnosis and effective treatment, as presented by ODEH and OLIVEN [9]. The present case demonstrates that cooperation between cardiologists and pulmonary specialists may result in a proper diagnosis and effective treatment of chronic cough following interdisciplinary investigations.

\section{REFERENCES}

1 Irwin RS. Complication of cough: ACCP evidence-based clinical practice guidelines. Chest 2006; 129: Suppl. 1, 54S-58S.

2 Morice AH, Committee Members. The diagnosis and management of chronic cough. Eur Respir J 2004; 24: 481.

3 Irwin RS, Baumann MH, Boulet LP, et al. Diagnosis and management of cough executive summary: ACCP evidence-based clinical practice guidelines. Chest 2006; 129: Suppl. 1, 1S-23S.

4 Mello CJ, Irwin RS, Curley FJ. Predictive values of the character, timing, and complications of chronic cough in diagnosing its cause. Arch Intern Med 1996; 156: 997-1003.

5 Kastelik JA, Aziz I, Ojoo JC, Thompson RH, Redington AE, Morice AH. Investigation and management of chronic cough using a probability-based algorithm. Eur Respir J 2005; 25: 235-243.

6 Canning BJ. Anatomy and neurophysiology of the cough reflex. ACCP evidence-based clinical practice guidelines. Chest 2006; 129: Suppl. 1, 33S-47S.

7 Zaborska B, Stec S, Flasinska K, Pilus A, Kulakowski P. Echocardiography and tissue Doppler imaging in assessment of haemodynamics in patients with idiopathic premature complexes. Pol Merkuriusz Lek 2005; 20: 302-304.

8 Nimii A, Kihara Y, Sumita Y, Okano Y, Tambara K, Fujita M. Cough reflex by premature ventricular contractions. Int Heart J 2005; 46: 923-926.

9 Odeh M, Oliven A. A man who coughed for 15 years before a doctor took his pulse. Lancet 1996; 348: 378 .

10 Zhou J, Scherlag B, Yamanashi W, et al. Experimental model of simulating right ventricular outflow tract tachycardia: a novel technique to initiate RVOT-VT. J Cardiovasc Electrophysiol 2006; 17: 771-775.

11 Kerr A Jr, Derbes VJ. The syndrome of cough syncope. Ann Intern Med 1953; 39: 1240-1253.

12 Boudoulas H, Nelson SD, Schaal SF, Lewis RP. Diagnosis and management of syncope. In: Alexander RW, Schlant RC, Fuster V, et al., eds. Hurst's The Heart. 9th Edn. New York, McGraw-Hill, 1998; pp. 1059-1080.

13 Benditt DG, Samniah N, Pham S, et al. Effect of cough on heart rate and blood pressure in patients with "cough syncope". Heart Rhythm 2005; 2: 807-813.

14 Malkoff MD, Gomez CR, Myles G, Cruz-Flores S. Cerebrovascular hemodynamic inefficiency of premature ventricular contractions. Angiology 1996; 1: 51-56.

15 Takemoto M, Yoshimura H, Ohba Y, et al. Radiofrequency catheter ablation of premature ventricular complexes from right ventricular outflow tract improves left ventricular dilation and clinical status in patients without structural heart disease. J Am Coll Cardiol 2005; 45: 1259-1265.

16 Gerstenfeld EP, Dixit S, Callans DJ, Rajawat Y, Rho R, Marchlinski FE. Quantitative comparison of spontaneous and paced 12-lead electrocardiogram during right ventricular outflow tract ventricular tachycardia. J Am Coll Cardiol 2003; 41: 2046-2053.

17 Ito S, Tada H, Naito S, et al. Development and validation of an ECG algorithm for identifying the optimal ablation site for idiopathic ventricular outflow tract tachycardia. J Cardiovasc Electrophysiol 2003; 14: 1280-1286.

18 Stec S, Walczak F, Kulakowski P, Zaborska B, Rezler J, Kokowicz P. Ventricular bigeminy originating from right ventricular outflow tract - treated with RF ablation. Kardiol Pol 2003; 58: 505-510. 